\title{
Aborto y Parlamento: un estudio sobre Brasil, Uruguay y Argentina*
}

\author{
Maria Isabel Baltar da Rocha** \\ Susana Rostagnol*** \\ María Alicia Gutiérrez ${ }^{* \star \star *}$
}

\begin{abstract}
El presente trabajo desarrolla un estudio comparativo entre Brasil, Argentina y Uruguay sobre las legislaciones y las acciones de diversos actores en relación a la despenalización y/o legalización del aborto. El tema se instala en la agenda pública en los tres países a partir del proceso de democratización iniciado en la década de los 80 produciéndose diversas intervenciones tanto del movimiento de mujeres, como de las corporaciones médicas y otros actores sociales en el transcurso de la década del 90 y los primeros años del Siglo XXI. En ese sentido se han trabajado las distintas intervenciones en el ámbito legislativo (tanto Cámara de Senadores como de Diputados), así como estrategias de políticas públicas implementadas desde los estados, con el monitoreo y seguimiento del movimiento de mujeres, para paliar una situación gravísima de violación de derechos humanos. El incumplimiento de los derechos, consagrados en los cuerpos constitucionales, afecta a las mujeres, especialmente las más vulnerables, en relación al derecho a la salud y la equidad. Se analizaron además los avances y retrocesos visualizados en el transcurso del período a estudiar, así como la importancia de mantener el tema en la agenda pública y en los medios masivos de comunicación que han cumplido un papel importante, tanto promoviendo, como deslegitimando el logro del derecho al aborto.
\end{abstract}

Palabras-clave: Aborto. Acción parlamentaria. Políticas públicas. Movimiento de mujeres. Actores sociales.

\section{Introducción}

La cuestión del aborto es un tema importante de la salud reproductiva y de los derechos humanos que atraviesa la casi totalidad de los países de América Latina y el Caribe y que afecta, de modo acentuado, a las mujeres más pobres. Se ponen en evidencia las condiciones de desigualdad de las relaciones sociales, expresándose en enfermedades y muertes relacionadas con la reproducción que podrían ser evitadas.

\footnotetext{
* Trabalho apresentado no III Congresso da Associação Latino-Americana de População, realizado en Córdoba, Argentina, de 24 a 26 de setembro de 2008.

** Pesquisadora do Núcleo de Estudos de População - Nepo, Universidade Estadual de Campinas - Unicamp, e professora colaboradora do Programa de Pós-Graduação em Demografia da mesma universidade. Assistente de pesquisa, Tânia Margatto. Apoio CNPq.

*** Professora do Instituto de Antropologia (Faculdade de Humanidades y Ciências da Educação), Universidade da República, e da Rede Uruguaia de Autonomias.

${ }_{* \star \star \star}$ Professora e pesquisadora da Faculdade de Ciências Sociais, Universidade de Buenos Aires e Foro pelos Direitos de Reprodução. Diretora do UBACYT S 409 Vozes polifônicas: sexualidades e identidades de gênero.
} 
El problema del aborto inseguro, derivado de su clandestinidad, practicado por personas sin la necesaria capacitación y/o en ambientes que no presentan un mínimo de condiciones sanitarias, conforme señala el Programa de Acción de la Conferencia Internacional de Población y Desarrollo (1994), coloca en la agenda el debate sobre la legalización de esa práctica.

Sólo en América Latina y el Caribe se realizan unos 3.700 millones de abortos inseguros, lo que representa una proporción de 32 abortos por cada 100 nacidos vivos y una tasa de 29 abortos por 1.000 mujeres de 15 a 44 años (GRIMES et al., 2006).

A pesar de las dificultades para constatar estadísticamente la práctica del aborto, dada su condición ilegal, las informaciones respecto a los países en que se centra nuestro trabajo también evidencian índices elevados. Respecto a Brasil, en un estudio a partir de los registros hospitalarios de internamientos por aborto, se estima el número de 1.054.242 abortos inseguros, correspondiendo a la razón de 30 abortos por 100 nacidos vivos y a la tasa de 20.7 por 1.000 mujeres entre 15 y 49 años, en el 2005, según los datos de una investigación que toma los registros hospitalarios de internamientos por aborto incompleto (MONTEIRO; ADESSE, 2006). En Uruguay las estimaciones alcanzan a 33.000 anuales en el 2002, esto representa un 38,5 de los embarazos (total de nacimientos, más abortos). En una población donde hay poco más de 800.000 mujeres en edad fértil, esta cifra supone 90,4 abortos diarios (SANSEVIERO, 2003). La tasa de abortos llega a 39 abortos por cada mil mujeres entre 15 y 44 años, siendo una de las tasas más elevadas de América Latina. Lo mismo puede decirse en relación a Argentina. En una investigación basada en estadísticas hospitalarias por complicaciones post-aborto, se estima la ocurrencia de 460.145 abortos inducidos en el año 2000 , equivaliendo a la razón de 66 abortos por 100 nacimientos, y una tasa de 50,4 por mil mujeres de 15 a 49 años (CEDES, 2007, CHECA; ROSENBERG, 1996)

No por casualidad, el tema fue objeto de discusiones y decisiones en las conferencias de las Naciones Unidas de los años 90, principalmente la de Población y Desarrollo (Cairo 94), en la que se estableció que el aborto es un asunto de salud pública, y la de la Mujer (Beijng 95), donde se propone una revisión de la legislación punitiva del aborto. A partir de los procesos de democratización de los años 80 , el tema integra el debate político en muchos países de la región. Del mismo modo, se incorpora al debate integrando las agendas relativas al Estado y la sociedad civil.

La discusión sobre la legalización del aborto ocurre, de este modo, en varias esferas políticas y públicas de la sociedad y del Estado, siendo el Parlamento un espacio privilegiado para esta finalidad. Esto se debe a que en los países con democracia representativa, como los nuestros, se trata de la principal institución responsable de la elaboración y proposición de leyes. En el presente artículo, dadas sus similitudes, pero también importantes diferencias, desarrollaremos una perspectiva comparada en tres países: Brasil, Uruguay y Argentina.

En los tres países el aborto es tipificado como crimen, constando en sus respectivos códigos penales. Hay algunas situaciones específicas, delimitadas en las respectivas legislaciones, en que esa práctica no es castigada, o existen eximentes y atenuantes a la pena, pero aún en estos casos no siempre es posible realizarlo. En los tres países, con distintos grados de intensidad, existen discusiones sobre el tema en el Parlamento, con la presentación de diversas propuestas de los congresistas, pero ningún cambio en el código penal fue realizado hasta ahora.

La idea que orienta el trabajo es que la redemocratización de Brasil, Uruguay y Argentina, a mediados de los años 80, representó un factor fundamental para hacer más visible la cuestión del aborto, creando condiciones para la ampliación del debate, para discusiones en el Parlamento y el Poder Judicial, así como para la elaboración de nuevas normas y políticas públicas y/o programas en el área de la salud. En el contexto de la redemocratización hubo un fortalecimiento de la sociedad civil y ha crecido su movilización en la búsqueda de conquistar y asegurar sus derechos de ciudadanía. En lo que se refiere a la cuestión del aborto, se 
han acentuado las acciones del movimiento feminista, principal actor comprometido con el cambio en la legislación relativa al asunto.

En cuanto al procedimiento metodológico, consideramos un objetivo común -en relación a los tres países- estudiar la cuestión del aborto en el ámbito del Poder Legislativo Nacional. Pretendimos construir un breve análisis para cada uno de los países y así crear condiciones para delinear un estudio comparado. El universo de la investigación es la discusión parlamentaria sobre el aborto, y en la medida de lo posible, la discusión política emprendida por los actores políticos y sociales que han estado participando de ese debate. Hay actores que comparecen en casi todo el periodo del estudio, como el movimiento feminista y la Iglesia Católica, y otros que han pasado a actuar posteriormente, como las entidades médicas e iglesias de denominación evangélica.

Finalmente, se trata, en gran parte, de un trabajo de base documental, teniendo como fuentes primarias proyectos de leyes y pronunciamientos del Poder Legislativo Nacional, además de documentos del gobierno y organizaciones de la sociedad civil, todos complementados con material de prensa. Como fuente secundaria, se utilizará la literatura especializada en el estudio sobre la cuestión del aborto en esos tres países.

\section{La polémica en el Congreso de Brasil}

Al construir un panorama sobre la cuestión del aborto en el Congreso Nacional, lo que nos despierta inmediatamente la atención es el énfasis dado a ese debate en algunos momentos de la historia reciente del país, sobretodo después de la redemocratización; así como la cantidad de proposiciones presentadas en esta casa legislativa (ROCHA, 1996, 2005, 2006; ROCHA; ANDALAFT, 2003; ROCHA; MORELL, 2006).

Siguiendo el recorrido histórico de esa discusión, es posible situar el primer proyecto de ley sobre el asunto en el año de 1949. Presentado por el diputado Monseñor Arruda Câmara, buscaba suprimir del Código Penal de 1940 los dos permisos legales relativos a la práctica del aborto, o sea, en las situaciones de riesgo de vida de la gestante y en el embarazo resultante de una violación. La presentación de ese documento, inmediatamente después de la apertura del Congreso, con el final del régimen del Estado Nuevo, y realizada por un sacerdote de la Iglesia Católica (institución que se va a constituir posteriormente como uno de los principales actores políticos en esa discusión), es el marco inicial del análisis de un debate que se ha estado desarrollando hasta el presente.

El debate, que abarca un periodo tan extenso, puede dividirse en tres etapas y algunas fases, definidas a continuación:

- etapa inicial y de calentamiento de la discusión: la primera fase abarca el periodo correspondiente al final de la década de los 40 hasta el inicio de la década de los 70 , con una discusión extremamente incipiente; la segunda fase cubre un periodo del inicio de la década de los 70 al inicio de la década de los 80 , con una participación todavía restringida de los actores políticos y sociales;

- etapa de transición, con intensificación del debate: se refiere a gran parte de la década de los 80 , con la participación más amplia de aquellos actores, inclusive en el ámbito de la Asamblea Nacional Constituyente, y se produce en el contexto de la transición política y después de la transición democrática, es decir, en el periodo que antecede y el que sucede a la caída del régimen militar;

- $\quad$ etapa de consolidación del debate: la primera fase abarca las dos legislaturas de la década de los 90, con varias iniciativas de parlamentarios identificados con las ideas del movimiento feminista, así como algunas iniciativas y muchas protestas de congresistas vinculados a religiones; la segunda fase se extiende desde final de la década de los 90 hasta la actualidad, con el debate ya consolidado en el Parlamento, y -al contrario de la fase anterior- con énfasis en la participación de los 
sectores vinculados a religiones, pero con algunas iniciativas y muchas protestas de los congresistas cercanos al movimiento feminista. En esa etapa se amplía la participación de actores políticos y sociales, aliándose de alguna manera, a esas dos principales tendencias.

En todo el periodo analizado fueron presentadas 109 proposiciones, incluyendo proyectos de ley, proyectos de decreto legislativo y propuestas de enmienda constitucional (información hasta el 31 de mayo de 2008). Iniciativas que han tenido una gran participación de la Cámara de Diputados, una menor participación del Senado Federal y aún menor del Poder Ejecutivo. Hasta el inicio de los años 90, fueron presentadas 31 proposiciones y en este conjunto la mayor parte reflejaba una posición de alguna manera contraria a permitir la práctica del aborto, aunque a partir de 1965 ya aparecían proyectos de ley pioneros, favorables a la despenalización y/o legalización del aborto.

Entre las propuestas con una visión crítica a esa despenalización y/o legalización, se puede decir que la única fue aprobada en 1979, una propuesta que también trataba sobre planificación familiar y control de la natalidad. Es necesario señalar, sin embargo, que esa propuesta no se ubicaba en el centro del debate sobre la cuestión del aborto, sino sobre planificación familiar. En realidad, suprimía de la Ley de las Contravenciones Penales de 1941 la referencia a la anticoncepción, pero mantenía en esta ley el dispositivo sobre la prohibición de divulgación de la práctica del aborto, es decir, "el anuncio de proceso, sustancia o objeto destinado a provocar aborto" (art. 20), actualizando la multa correspondiente.

También en ese amplio periodo anterior a la década de 1990, cabe señalar que el tema aborto provocado fue considerado una de las cuestiones polémicas de la Asamblea Nacional Constituyente-1987/1988-y exactamente por este contenido polémico terminó por no constar de la nueva Carta Magna. El tema fue incluso objeto de propuestas de la sociedad presentadas al Parlamento, las denominadas enmiendas populares. En el contexto de la Constituyente aparecían serias divergencias en relación con esa materia, concernientes a la defensa de la vida desde el momento de la concepción o, diferentemente, a la defensa del derecho de la mujer a decidir sobre ese asunto. La primera posición tenía influencia de la jerarquía de la Iglesia Católica y de las religiones de denominación evangélica, y la segunda se inspiraba en los movimientos feministas y de mujeres. Esos actores políticos y sociales tendrán cada vez mayor participación en ese debate en los años 90 y en la década actual, como se podrá observar por el contenido de las proposiciones presentadas.

La polémica en el Congreso Nacional, intensificada en los años 80, tuvo aún mayor énfasis en las dos legislaturas de los años 90 y en las tres de la década actual -lo que se denominó, anteriormente, primera y segunda fase de la etapa de consolidación del debate. Las fases se refieren, por lo tanto, a las legislaturas 1991-1995 y 1995-1999, y después a las de 1999-2003, 2003-2007 y 2007-2011, ésta última en progreso. En total han sido presentadas 78 propuestas, lo que representa alrededor de un $72 \%$ del total enviado hasta ahora, y sus dos fases corresponden al predominio de diferentes tendencias en relación al tema, con significativas excepciones.

En las dos legislaturas de los años 90, han sido presentadas en el Parlamento 23 propuestas. Entre ellas, la gran mayoría era, de alguna manera, favorable al permiso de aborto, cerca de un $70 \%$, existiendo así un vuelco en la discusión en relación con el amplio periodo anterior. Sin embargo, ese cambio que ocurrió con nitidez en la primera legislatura de esa década, ya empieza a modificar su perfil en la segunda legislatura mencionada.

En esta fase, que abarca las primeras legislaturas, después de la promulgación de la Constitución de 1988, predominaron las iniciativas de congresistas identificados con las ideas del movimiento feminista. Incluso se aprueba un proyecto de ley de alguna manera favorable al aborto, pero que no se sitúa en el centro del debate, ya que es una propuesta referida a derechos laborales. Ésta determina que no se configura como falta al trabajo la ausencia de la empleada 
debido a un aborto, independientemente de la legalidad de esta práctica. En realidad, se trata del segundo de los dos únicos proyectos aprobados en todo el periodo estudiado, y ambos, aunque con tendencias distintas, pasan al margen del núcleo central de la cuestión, es decir, la aceptación o no de la práctica del aborto como un derecho de la mujer.

Es importante destacar también, en relación con esa fase, el intenso debate durante la discusión del proyecto sobre la atención al aborto legal en el Sistema Único de Salud, que fue aprobado en dos comisiones en la Cámara, se convirtió en objeto de una Comisión General en su pleno, pero no tuvo condiciones de ser votado (se encuentra todavía en esa casa legislativa). Por otro lado, cabe señalar que han sido allí rechazados, también en un clima de vehemente debate, dos proposiciones contrarias al derecho al aborto. En ese caso, una propuesta de enmienda constitucional sobre la inclusión de la idea de la defensa de la vida desde el momento de la concepción, y por fin un proyecto de decreto legislativo sobre el intento de suspender la norma técnica del Ministerio de la Salud, que incluye la atención al aborto previsto en ley. Además de los cuatro documentos destacados, hay todavía una quinta propuesta, esta vez sobre la despenalización del aborto en determinadas circunstancias: el proyecto de ley $1135 / 91$. Pese a que fue presentado en 1991, se convirtió en centro de discusión sobre todo en 2005 , y después en 2007 y 2008; por eso decidimos tratarlo separadamente al final de este ítem del trabajo

En las tres legislaturas de la década actual se presentaron 55 proposiciones. Al contrario de la fase anterior, la mayor parte (cerca de un $78 \%$ ) es de alguna manera contraria al permiso de la práctica del aborto, a saber: la casi totalidad de las proposiciones de la primera legislatura citada, 1999-2003, y la mayor parte de la segunda, 2003-2007, y nuevamente la casi totalidad de los proyectos de la legislatura en progreso.

En este momento, a pesar del gran número de propuestas y del amplio abanico de aspectos presentados, sobretodo provenientes de los parlamentarios contra- rios a la legalización del aborto (en general vinculados a religiones cristianas), el debate en el Parlamento fue relativamente más restringido que en la fase anterior; es decir, si consideramos las discusiones en las comisiones técnicas, la realización de audiencias públicas y la repercusión en la sociedad. De ese conjunto de proyectos, diez han sido apreciados en alguna comisión, y de ésos, cuatro se encuentran archivados y seis en trámite, estos últimos presentados más recientemente. Entre esos diez proyectos, sin embargo, vale subrayar dos que están más directamente vinculados al núcleo del debate sobre la cuestión del aborto, a saber: un proyecto de ley que suma al código penal un nuevo permiso para la situación de la interrupción del embarazo, en caso de anencefalia, que fue aprobado en la Comisión de Seguridad Social y Familia en 2005, y otro proyecto de decreto legislativo sobre la suspensión de la referida norma técnica del Ministerio de la Salud, que fue rechazado en la referida comisión de la Cámara de Diputados en 2007.

Aunque las proposiciones de esta fase hayan despertado la discusión de las diferentes tendencias, no han llegado a causar el debate reñido de la década pasada, por lo menos hasta el momento presente. En realidad, la gran obstinación de ese debate se está produciendo en relación al PL 1135/91, que será tratado a continuación.

Ese proyecto de ley dispone sobre la supresión del artículo 124 del código penal, referente a la incriminación de la mujer que provoca el aborto o permite que alguien se lo provoque. Durante alrededor de 15 años muchos otros proyectos fueron anexados a éste, así como en el inicio de la presente década recibió un dictamen de su relatora que ampliaba en mucho las posibilidades de realización del aborto. En el año de 2005, en el contexto de la discusión sobre la presentación de un anteproyecto de la Comisión Tripartita al Congreso Nacional, se acordó políticamente que esa parlamentaria incorporaría a su proyecto sustitutivo el anteproyecto de la Tripartita, creando condiciones para que fuera apreciado aún durante aquel año. 
La Comisión Tripartita de Revisión de la Legislación Punitiva del Aborto fue constituida por la Secretaría Especial de Políticas para las Mujeres en 2005, como consecuencia de los resultados de la I Conferencia Especial de Políticas para las Mujeres, que propuso la revisión de la legislación punitiva del aborto. Formada por integrantes de los poderes ejecutivo y legislativo y de la sociedad civil, y con fuerte influencia del movimiento feminista, esa comisión formuló un anteproyecto de ley para presentarlo en el Congreso.

El proyecto sustitutivo, o como pasó a ser llamado, de la Comisión Tripartita, versa sobre la legalización del aborto, incluyendo la atención a través del Sistema Único de Salud y determinando su cobertura por los planes privados de asistencia a la salud. Establece el derecho a la interrupción del embarazo, y su ejercicio implica que sea realizado por un médico y con el libre y claro consentimiento de la gestante. Esa interrupción queda asegurada en cualquier circunstancia, en primer lugar, durante las 12 semanas de gestación; respetadas las implicaciones mencionadas, queda también asegurada hasta las 20 semanas, cuando el embarazo es resultado de un crimen contra la libertad sexual; y por fin, queda garantizada en situaciones de grave riesgo para la salud de la gestante y de malformación congénita incompatible con la vida o grave e incurable enfermedad en el feto.

Ese proyecto sustitutivo no tuvo condiciones políticas para ser votado en 2005, por la controversia que generó, ni siquiera en 2006, año electoral. En 2007, la relatoría del PL 1135/91 se lo entregó a un parlamentario de otra tendencia que presentó un dictamen contrario a éste y a un proyecto sobre la despenalización del aborto a él anexado. En mayo de 2008, los dos fueron rechazados en la Comisión de Seguridad Social y Familia y en julio (también con dictamen contrario), en la Comisión de Constitución y Justicia y de Ciudadanía. Objeto de intenso debate en las audiencias públicas convocadas para discutirlos y en las reuniones de esas comisiones, todo indica que aún será discutido en el pleno de la Cámara de Diputados.
El cuadro aquí dibujado, que refleja un juego político repleto de meandros, pero sin presentar cambios sustanciales en la legislación, está asociado a la acción de los actores políticos y sociales y su influencia directa o indirecta en el Congreso Nacional.

\section{El debate legislativo en Uruguay}

El aborto es delito y está penalizado por la ley $\mathrm{n}$ - 9.763 del 28 de enero de 1938. La misma ley presenta atenuantes y eximentes a la pena, siempre y cuando el aborto haya sido realizado por un médico con consentimiento de la mujer, dentro de las primeras 12 semanas por una de las siguientes causas: peligro para la salud o vida de la mujer; embarazo consecuencia de una violación; penuria económica u honor.

En el período 1934-1938 el aborto estuvo despenalizado. Según la historiadora feminista Graciela Sapriza (2002), esto habría sido más el resultado del peso de las corrientes eugenésicas que consecuencia de ideas liberales. En 1938 es recriminalizado con la ley 9.763 , vigente hasta hoy. Esta acción debe entenderse integrando un proceso de biopolítica, usando la terminología foucauldiana; ya que estuvo de la mano de una serie de otras acciones que resultaban en la medicalización de la sociedad como mecanismo de control/gobernabilidad.

La Ley del 38 exige que el aborto sea realizado por médicos y con el consentimiento de las mujeres para que puedan aplicarse los atenuantes y eximentes a la pena; quedándoles expresamente prohibido a las parteras. Inmediatamente de promulgada la ley, la ordenanza 179 y los decretos complementarios "constituyen una secuencia de normas que pueden interpretarse como 'un diálogo' entre la praxis social y la tendencia reguladora de disciplinamiento y medicalización del aborto" (SANSEVIERO, 2008, p. 137), ya que éstas especifican el papel de los médicos en los abortos dentro de plazo y causas, y la voluntad de la mujer estipulados para ser considerados dentro de los atenuantes y eximentes a la ley; asimismo define el carácter obligatorio de la denuncia epidemiológica, junto a la confidencialidad sobre la identidad de la mujer. El análisis de 
los actos de gobierno inmediatamente posteriores a la promulgación de la ley permiten pensar que el aborto voluntario constituye "un hecho adjetivo, siendo su preocupación central la regulación de la práctica" (SANSEVIERO, 2008, p. 17).

Con el avance del siglo XX, se agudizaron las restricciones al aborto, volviéndose más secreta su práctica. Las denuncias epidemiológicas dejaron de lado la confidencialidad de la identidad de las mujeres. En los 90 se hizo frecuente que los médicos denunciasen mujeres que acudían a los servicios hospitalarios con complicaciones post-aborto (ROSTAGNOL, 2003; SANSEVIERO, 2003), aunque difícilmente esto tuviera consecuencias judiciales. El contexto cultural uruguayo ha sido siempre de 'condena, tolerancia y negación' para las prácticas del aborto voluntario. Ese es el contexto en que se tienen lugar los sucesivos debates parlamentarios sobre proyectos de ley que incluyen la legalización del aborto.

En base a un trabajo previo (ROSTAGNOL, 2003) se presenta de manera sucinta las propuestas de cambios legislativos que despenalizan el aborto. Tomando un lapso ligeramente mayor al propuesto, se puede establecer los siguientes momentos y actores:

- 1978-79. Iniciativa de legalización emanada, en plena dictadura, desde la Jefatura de Policía de Montevideo. Se crea por iniciativa del Ministro del Interior de la época una Comisión integrada por miembros de los Ministerios de Justicia e Interior que elabora un proyecto muy articulado estableciendo el aborto "por voluntad de la mujer dentro de las primeras 12 semanas de gestación". Las jerarquías militares discuten el proyecto y lo descartan;

- 1984-85. Las organizaciones de mujeres presentan ante la Concertación Nacional Programática (que articula la salida de la dictadura) el tema del aborto para ser discutido. Por el funcionamiento consensual de esa instancia, el tema no es aceptado;
- 1985. Primera iniciativa parlamentaria post-dictadura para volver a la despenalización de 1934 presentada por diputados del Partido Colorado: no prospera el debate sobre el tema;

- 1993-94. Proyecto de Ley de "Regulación de la voluntaria interrupción de la gravidez" (presentado por el diputado del Frente Amplio Rafael Sanseviero, y elaborado en colaboración con la abogada feminista Graciela Dufau). Establece el "derecho de toda mujer a decidir sobre la interrupción de la gravidez dentro de las 12 semanas" y a poder acceder a abortos más allá de ese lapso, en caso de existir motivos de salud u otros. Al tiempo que se consagra el derecho a la objeción de conciencia por parte de los médicos, establece que los servicios de salud están obligados a contar en su plantilla con profesionales dispuestos a brindar el servicio de aborto. Es aprobado por unanimidad en la Comisión de Bioética de la Cámara de Diputados y no llega a discutirse en el plenario de la Cámara;

- $\quad$ 1998. Se presenta el proyecto del 93 con leves modificaciones en la exposición de motivos, presentado por el Frente Amplio;

- 2002-2004. Es aprobado en diciembre de 2002 por la Cámara de Diputados el proyecto de ley de Defensa de la Salud Reproductiva que recoge los contenidos y la articulación del de 1993-94. Un año y medio después el Senado vota en contra de su promulgación (15 a 13 votos), esgrimiendo algunos legisladores la falta de oportunidad política para votar a favor, ya que se trataba de un año electoral. Este proyecto incluye la incorporación de educación sexual en la enseñanza formal y garantía de acceso a métodos anticonceptivos. En 2001 un aumento en las muertes 
de gestantes por complicaciones post-aborto, llevó a parte de la comunidad médica -reunida en Iniciativas Sanitarias- a la búsqueda de alternativas que permitieran disminuir la mortalidad. El problema en sí mismo, conjuntamente a la presión social, hace reaccionar a la bancada femenina que desarchiva el proyecto de ley, desempeñando un rol protagónico en el Parlamento. Es destacable el papel desempeñado por la Coordinadora Nacional de Organizaciones por la Defensa de la Salud Reproductiva que, liderada por organizaciones feministas, logró una base amplia y plural de apoyo durante los dos años que duró la discusión legislativa.

La preocupación de los médicos convertida en acción tuvo tres consecuencias en los años siguientes: la primera, situó a los médicos como un actor clave en las demandas por la legalización del aborto; en segundo lugar, permitió que se desarrollaran políticas públicas que atendieran el aborto inseguro, tensando la ley vigente; finalmente, y como consecuencia de lo anterior, habilitó hablar del aborto en los ámbitos cotidianos, es decir, las mujeres pueden manifestarle a su ginecólogo su intención de abortar, sin que esto tenga consecuencias legales para ella. En el 2004, una vez fracasado el intento de legalización del aborto, el Ministerio de Salud Pública aprueba la ordenanza 369 que refiere a la atención pre y post aborto, dejando fuera la instancia específica del aborto. El protocolo permite disminuir la posibilidad de morbimortalidad. A su vez, la difusión del misoprostol para provocar abortos también ha disminuido las complicaciones serias post-aborto. Resulta paradójico que luego de reafirmada la penalización del aborto (al haber votado en contra del proyecto de ley) se implemente una normativa que ayuda a las mujeres a abortar en mejores condiciones y que reafirma la confidencialidad hacia la usuaria.

- 2007-2008. Nuevamente un proyecto de ley, con muy pocos cambios respecto al anterior, es presentado para su discusión en el Senado a mediados de 2007. Importa realizar un repaso en relación a la aplicación de las leyes y normativas vigentes a fin de valorar las discusiones. Prácticamente, sólo se han realizado procesamientos con prisión por el delito de aborto cuando se produce la muerte de la mujer. De acuerdo a la información presentada por Sanseviero (2003), entre 1989 y 1997 hubo un promedio de 13,7 procesamientos al año por delito de aborto. En 2000 y 2001 el promedio asciende a 16, lo que significa que los procesamientos corresponden a $0,04 \%$ de los abortos ocurridos de acuerdo a la estimación de 33.000 abortos por año. Esto muestra que se trata de una práctica extendida y tolerada. Sin embargo, es preciso señalar que son prácticamente nulos los abortos realizados dentro de los eximentes y atenuantes que establece la ley. Hasta el 2007 se había registrado un solo caso de aborto, son más comunes los casos por motivo de violación; sin embargo, Rostagnol (2007) registra el caso en 2006 de una menor de edad violada con la denuncia policial realizada, a quien se le propuso realizarse el aborto de manera clandestina, porque el camino burocrático era muy largo.

Esto da cuenta que en el período considerado para el presente análisis la ley discutida una y otra vez en el Parlamento es una "ley en desuso", según expresión del jurista Langon (1979). Este aspecto introduce un campo de interrogantes sobre cuál es el verdadero tema o problema en discusión, ya que resulta por lo demás asombroso la ausencia de menciones al incumplimiento de la ley que penaliza el aborto. Esto tuvo su 
pico cuando en 2007 el procesamiento sin prisión de una mujer, como resultado de la denuncia de un médico, motivó que un grupo denominado "ciudadanas y ciudadanos" llevara adelante una campaña de autoinculpación del delito de aborto que en un mes contó con 9000 firmas incluyendo varios ministros. Como respuesta a la presión ejercida por "ciudadanos y ciudadanas", la corporación médica realiza una declaración en cual establece que "el secreto médico no es una opción, sino una obligación para los médicos y el equipo de salud", con lo cual queda garantizada la confidencialidad para con la mujer en situación de aborto. Este estado de cosas hace pensar en la existencia de "una fuerte 'disociación' entre la práctica social y el reflejo de esa práctica en la sociedad política" (SANSEVIERO, 2008, p. 131).

La presión social resultante del movimiento de autoinculpación hizo que el Senado tratase el proyecto de ley que tenía entre manos. En noviembre de 2007 lo aprueba. Pasa luego a la Cámara de Diputados, donde se aprueba con modificaciones por lo que debe volver al Senado. Éste finalmente aprueba el proyecto de ley el 11 de noviembre de 2008. Inmediatamente, el Presidente Tabaré Vázquez veta la ley. La Asamblea General no logra levantar el veto, por lo que el aborto continuó siendo un crimen.

En las sucesivas discusiones parlamentarias (Senado, Diputados, nuevamente el Senado), los argumentos esgrimidos por los legisladores contrarios a la legalización se caracterizaron por centrarse en generalizaciones abstractas, generalmente referidas al inicio de la vida -a la que se homologaba con el carácter de 'persona' otorgado al cigoto y embrión-, en base a fundamentos tanto religiosos como científicos, y a la preocupación por la baja tasa de natalidad que tiene el país. Por su parte, los argumentos de los legisladores favorables a la legalización del aborto se basaban en aspectos epidemiológicos referidos a la morbimortalidad, como consecuencia de abortos inseguros; a los derechos de las mujeres, en algunos casos desde una perspectiva de género. En términos generales es posible observar una clara diferencia en el 'sujeto de derecho' al que se refieren unos y otros (ROSTAGNOL, 2008).
En el proceso de estas discusiones legislativas, la sociedad civil amplió aún más su base. Los grupos feministas pasaron a compartir con grupos de derechos humanos y de jóvenes y, aunque desde otro lugar, con la corporación médica las actividades de abogacía. Las encuestas de opinión daban un $63 \%$ de la población favorable a la aprobación del proyecto de ley de Defensa del Derecho a la Salud Sexual y Reproductiva en el momento en que el Presidente veta la ley que despenalizaba el aborto.

\section{La discusión parlamentaria en Argentina}

"Las leyes no solamente sancionan y determinan conductas sino, sobre todo, expresan el modelo de país que queremos. De manera asombrosa y alarmante se sancionan y legitiman conductas y estilos de vida antinaturales" estas declaraciones pertenecientes al "guardián de la heterosexualidad" Mario Maulión, Arzobispo de Paraná, son una muestra de las concepciones conservadoras, fundamentalistas, respecto a la relación familia, estado y orden social. En este marco ideológico local es donde se despliegan las estrategias por la despenalización/legalización del aborto.

Diversos antecedentes legales han ubicado la problemática del aborto en el campo de las restricciones. En 1919 se introducen formas de impunidad en la figura del aborto, tomadas del anteproyecto del código penal suizo, sancionadas por el Congreso Nacional por ley 11.179, vigente desde 1922. La copia realizada del anteproyecto suizo incluyó errores de redacción, que generaron discusiones doctrinarias sobre su alcance. El agregado de una coma en el inc. $2^{\circ}$ del art. 86, propuesto por la diputada Florentina Gómez Miranda en 1989 permitiría extender la no punibilidad del aborto por violación a todas las mujeres, y no sólo a las idiotas o dementes, como se interpreta usualmente este inciso (MINYERSKY, 2005).

El texto actual es tratado en el Capítulo I "Delitos contra la vida". Es el mismo capítulo que contiene otros tipos penales: homicidio, infanticidio, duelo, entre otros. El art. 86 refiere al aborto realizado por profesionales (médicos, cirujanos, parteras 
o farmacéuticos) y a las figuras impunes de aborto terapéutico, eugenésico y sentimental. Es de destacar que este artículo sufrió cuatro modificaciones y que ha sido uno de los que más discusiones ha provocado. En su segundo párrafo establece las dos situaciones en las que la interrupción del embarazo practicado por un médico, con el consentimiento de la mujer, no es punible: “... para evitar un peligro para la vida o la salud de la madre y si éste no puede ser evitado por otros medios...", y "si el embarazo proviene de una violación o de un atentado al pudor cometido sobre una mujer idiota o demente. En este caso, el consentimiento de su representante legal deberá ser requerido para el aborto".

La vigencia del texto original recrea la discusión histórica en torno a los abortos "no punibles", en especial aquel que suspende la gestación originada en una violación. La postura restringida (actualmente en vigencia) lo condena; interpretando que se refiere sólo a "la violación de una mujer idiota o demente". Los precedentes legislativos argentinos del código penal siempre tipificaron el aborto no previendo excepciones ni formas de impunidad. En la práctica, estos supuestos despenalizadores no se aplican, ya que en muchos casos los médicos se niegan a realizar los abortos sin contar con una autorización judicial, la cual generalmente sufre retrasos (DOMÍNGUEZ et al., 2004).

A partir del proceso de democratización iniciado en 1983, se han presentado 12 proyectos en ambas cámaras, con planteamientos de reformas, pero ninguno logró ser sancionado, quedando en las instancias de trámites parlamentarios burocráticos y sin tratamiento legislativo.

Dos de ellos derogaban las figuras de aborto no punible y cinco mejoraban su redacción; tres legalizaban el aborto; un proyecto lo despenalizaba y uno incorporaba al código penal la protección de la persona por nacer.

Los proyectos presentados desde el inicio de la transición democrática en 1983 hasta la actualidad enfocan cuestiones diversas y marcan desde qué Cámara se inició el proceso de presentación. Ninguno de ellos lograron trascender la presentación sin llegar a debatirse en comisiones y menos aun en los recintos, por lo tanto sigue vigente el Código de 1922.

\section{Cámara de Diputados}

- $\quad$ 1989. El proyecto presentado instaló el tema del aborto en los casos de violación en el debate público, proponiéndose su no incriminación.

- 2003 - Proyecto de Ley: modificación al Código Penal Sumario: Modificación al art. 86 (Aborto, Penalización para los profesionales implicados).

- 2003 - Proyecto de Ley: reconocimiento del derecho humano de la mujer a la interrupción de su embarazo en los casos permitidos por el Artículo 86. El estado nacional debe garantizar dicho derecho a través del sistema de salud pública.

- 2003 - Proyecto de Ley: modificación del artículo 86 del Código Penal, sobre la comisión del delito de aborto.

- 2003 - Proyecto de Ley: régimen de interrupción voluntaria del embarazo; modificación del código penal en lo relacionado al delito del aborto. Establece claramente los límites para que los médicos del servicio de salud pública, privada y obras sociales realicen los abortos establecidos por el código penal de forma informada y expeditiva, sin requerir la autorización judicial. Interpreta de manera amplia el concepto de salud, que incluye la salud psíquica y considera el embarazo por violación como un caso de peligro para la salud de la mujer, como así también en casos de fetos con comprobadas patologías incompatibles con la vida extrauterina. Se trata de que los médicos del sistema de salud público, privado, y las obras sociales, puedan realizar las intervenciones con la indicación médica como único requisito, sin 
que sea necesaria la autorización de un juez.

- 2004 - Proyecto de Ley: modificación sobre aborto no punible.

- 2004 - Proyecto de Ley: modificaciones sobre despenalización del aborto en casos específicos.

- 2004 - Proyecto de Ley: consulta popular vinculante para determinar legalidad o ilegalidad del aborto.

\section{Cámara de Senadores}

- 1986, para casos de embarazos productos de una violación, incluía como figura atenuada en su pena, la del aborto por causa de honor. Con dictado favorable de comisión, fue considerada en el recinto, en el que se introdujeron tantas modificaciones, que al finalizar su tratamiento, el Senado Nacional en el año 1990, terminó aprobando un texto idéntico al actual código penal sin agregar las modificaciones propuestas.

- 2004 - Proyecto de Ley sobre despenalización del aborto para los casos de violación.

- 2004 - Proyecto de Ley sobre tipificación del delito de violación seguido de embarazo.

- 2004 - Proyecto de Ley sobre despenalización parcial del aborto, declarando no punible el aborto, si el embarazo proviene de la comisión de un delito contra la integridad sexual y si se ha diagnosticado médicamente la inviabilidad de vida extrauterina del feto.

- 2004 -Proyecto de Ley de despenalización del aborto para casos específicos: violaciones; situaciones en la que está en riesgo la vida de la mujer; imposibilidad de vida extrauterina del feto.

Todos los proyectos presentados por senadores se encuentran unificados en la Comisión de Justicia y asuntos penales a la que le corresponde dictaminar en relación al Código Penal, como así también respecto a toda disposición de carácter punitivo o represivo que contenga cualquier rama del derecho.

Puede observarse que tras un primer intento de colocar el tema en la agenda parlamentaria, inmediatamente después de restaurada la democracia, el Congreso argentino no discute el tema del aborto durante la década de los 90 , dado que la problemática del aborto -como los derechos sexuales y reproductivos- reaparecerán en el escenario político bajo la impronta de una coalición conservadora entre el gobierno Justicialista y la Iglesia Católica, que intenta, tanto a nivel nacional como internacional, promover las posiciones sostenidas por el Vaticano en materia de sexualidad y género (GUTIÉRREZ, 2003). En el proceso de la Reforma Constitucional de 1994 una alianza conservadora entre la jerarquía eclesiástica y el Partido Justicialista promovieron la introducción de una cláusula sobre el derecho a la vida desde la concepción, en el cuerpo constitucional, lo que provocó reacciones adversas especialmente en el movimiento de mujeres. La importante acción del Movimiento de mujeres permitió, pese a la fuerte presión de la Iglesia, que la cláusula no fuera incluida en el texto constitucional.

Tras las conferencias internacionales de El Cairo (1994) y de Beijing (1995) se establecieron estrategias conjuntas con legisladoras para intentar lograr una Ley Nacional de Procreación Responsable. Los avatares de esas estrategias llevaron a la conformación, desde el movimiento de mujeres de un grupo heterodoxo en términos de pertenencias institucionales y políticas que se denomino MADEL (Mujeres Autoconvocadas para decidir en Libertad). El "clima" logrado en Beijing y la importancia de las resoluciones finales inspiraron la necesidad de contar con una Ley de Salud Reproductiva. La presentación de un proyecto conjunto por un grupo de diputadas acompañadas por MADEL permitió poner en el debate público la necesidad de legislaciones y políticas públicas sobre la salud y los derechos sexuales y reproductivos (GUTIÉRREZ, 2004b). En 1998, durante 
la presidencia de Carlos Menem, se estableció el 25 de marzo como "día del niño por nacer".

Finalmente, la Ley aprobada en la Cámara de Diputados, denominada Ley de Salud Reproductiva y Procreación Responsable pasó a la Cámara de Senadores con una oposición más fuerte y estructurada por la composición mayoritaria del partido oficialista. Allí estuvo para su tratamiento durante dos años hasta que venció el plazo y quedó sin efecto su sanción. Recientemente, en 2002 y con muy escasa participación del movimiento de mujeres se logró la Ley $\mathrm{Na}$ cional de Salud Reproductiva y Procreación Responsable, consensuada su articulación entre legisladoras y representantes de la iglesia católica, la cual no hace mención al aborto.

Por otra parte, tampoco hubo avances en la aplicación del Plan de Acción de la Conferencia de El Cairo y de su revisión $($ Cairo +5$)$ relativos a la capacitación y adecuación de los servicios de salud para atender las complicaciones del aborto (CELS; CLADEM; FEIM; ISPM, 2002). La misma situación sin grandes variantes se evidenció en las reuniones de Cairo +10 y Beijing +10 .

Con el cambio de gobierno a partir del 2003 aparecen nuevamente proyectos de ley que tratan el tema del aborto. A principios del año 2005, el Ministro de Salud de la Nación, Dr. Ginés, realizó declaraciones públicas a favor de la despenalización del aborto por razones de salud pública, lo que facilitó nuevas acciones de políticas públicas y a su vez un respaldo a la acción del movimiento de mujeres de la 'Campaña Nacional por el derecho al aborto seguro, gratuito y libre'. De la convicción del ministerio surgieron las directivas para la atención posaborto y la consejería, en el año 2007, pero aun no es aplicada en la totalidad de los servicios de salud (GUTIÉRREZ, 2007).

Es en estas condiciones de ilegalidad que el movimiento de mujeres ha desplegado sus estrategias de lucha en relación a la problemática del aborto, denunciando, impulsando y acompañando distintas propuestas parlamentarias.

\section{Algunas consideraciones sobre la cuestión del aborto en Brasil, Uruguay y Argentina}

Al concluir este trabajo, recuperamos su idea central y la confirmamos, o sea, la redemocratización en los tres países estudiados representó un factor fundamental para hacer más visible la cuestión del aborto, creando condiciones para la ampliación del debate en la sociedad, para discusiones en el Parlamento y el Poder Judicial, así como para la elaboración de nuevas normas y políticas públicas y/o programas en el área de la salud. También hubo repercusión de los resultados de las Conferencias de las $\mathrm{Na}$ ciones Unidas, sobre Población y Desarrollo y sobre la Mujer, en las discusiones políticas y en las políticas públicas de esos países.

Para este estudio nos detuvimos, principalmente, en el análisis de los respectivos parlamentos nacionales y en la articulación de éste con otros actores políticos y sociales. Resaltamos la actuación del movimiento feminista y de algunos segmentos de profesionales del área salud comprometidos con los cambios legislativos, así como los partidarios del mantenimiento de la ley restrictiva o de su endurecimiento, generalmente vinculados a algunas iglesias cristianas y a los movimientos pro-vida. Constatamos la importancia del debate parlamentario, más intenso y polarizado dependiendo del país o de la ocasión, como así también que no hubo cambios legislativos o cuando ocurrieron no fueron substanciales.

Las observaciones finales referidas a cada país, presentadas a continuación, van a recuperar esos aspectos, así como el dinamismo de la discusión sobre el aborto en el campo de los derechos sexuales reproductivos. Al mismo tiempo, van a señalar algunos cambios, aún con contradicciones, en el ámbito de las políticas públicas -cambios especialmente en el área de la atención de la salud, que muestran la búsqueda de estrategias políticas para enfrentar la cuestión de la ilegalidad del aborto y de sus consecuencias para la salud y la vida de las mujeres.

En lo que se refiere a Brasil, durante todo el periodo estudiado no hubo altera- 
ciones en el Código Penal en relación al aborto, como ya se ha visto. Cambios que despenalizasen y/o legalizasen el aborto, ni tampoco que ampliasen las posibilidades de práctica de la interrupción de la gestación en el país, o por el contrario, cambios que eliminasen los permisos legales vigentes. En ese contexto de la legislación, el aborto inseguro sigue siendo ampliamente practicado con graves consecuencias, sobretodo para las mujeres más pobres.

Las principales fuerzas políticas que vienen actuando en el parlamento respecto a la cuestión, generalmente tienen concepciones distintas sobre las relaciones de género, sexualidad y reproducción -con sus respectivos compromisos políticos- lo que lleva a una polarización de la discusión y la consecuente ausencia de modificaciones sustantivas en la ley. El debate refleja y potencia la tensión política entre, por una parte el movimiento feminista y de mujeres, organizaciones de salud sobretodo en el área de ginecología y obstetricia y demás aliados por la despenalización y/o legalización del aborto, y por otra parte la jerarquía de la Iglesia Católica, diversas iglesias evangélicas y los movimientos pro-vida, este último conjunto con intensa acción conservadora en la presente década (SANTIN, 2005; GOMES, 2007). Los principales partidos políticos tienen dificultades para manifestarse formalmente en relación con la cuestión, una vez que el asunto es generalmente motivo de gran controversia entre sus integrantes.

A pesar de la ausencia de transformaciones en el Código Penal, hay un debate de ideas sobre el tema y en lo que refiere a esa cuestión se han dado algunas modificaciones en el ámbito de la sociedad, de los poderes legislativos, judicial y ejecutivo. Son exactamente modificaciones en la esfera del poder ejecutivo y de las políticas públicas en relación al aborto las que se pretenden destacar, brevemente, al cerrar esta parte del trabajo. Esos cambios -aunque parcialesdifícilmente serían implementados en un país en que no hubiese una sociedad civil activa en un contexto de democracia política.

Nos estamos refiriendo a los servicios de asistencia al aborto no penado por la ley, es decir, el servicio de aborto legal. En primer término han sido constituidos en algunos municipios, estados y universidades, de acuerdo a normas específicas, a partir de la segunda mitad de los años 80 , y seguidamente, a partir del final de los años 90 , extendidos para todo el país, después de su reglamentación en la esfera del Ministerio de la Salud. En ese caso, teniendo como referencia la Norma Técnica para Prevención y Tratamiento de los Agravios Resultantes de la Violencia Sexual contra Mujeres y Adolescentes, cuya primera versión es de 1998 y la segunda, revisada y ampliada, de 2005 (TALIB; CITELI, 2005).

Es posible afirmar que las mujeres feministas, al lado de profesionales de salud comprometidos con el tema, han desempeñado un rol fundamental en la formulación de los documentos y la implementación de esos servicios, a pesar de las objeciones de sectores religiosos, incluso en el Congreso Nacional. La atención al aborto legal en los hospitales públicos y universitarios representa, de hecho, un cambio importante en el tratamiento del problema en el país, aunque no supere los límites de la legislación que restringe el derecho al aborto, y en consecuencia, los derechos sexuales y reproductivos, así como presente algunos problemas concernientes a la cantidad y la concentración de las unidades hospitalarias, y a veces a la calidad de la atención a la salud.

En Uruguay, tal como se ha visto, ya se cumplieron 70 años de vigencia de la ley que penaliza el aborto. Son también 70 años en que los abortos son tolerados y aceptados por la sociedad. Durante el periodo se han sucedido momentos de mayor permisividad, junto a otros en que aumenta la represión fundamentalmente a las clínicas clandestinas. A la escisión entre la legislación y la práctica social, se agregan algunas contradicciones entre las normativas referidas al aborto.

La ordenanza 369/2004 del Ministerio de Salud Pública propone un asesoramiento a mujeres que consideren la posibilidad de interrumpir su embarazo. Se basa en una estrategia de reducción de riesgos y daños. Consiste en una consulta inicial 
donde, además de realizar una ecografía para verificar la edad gestacional, se informa sobre la manera de procesar un aborto con reducción de riesgos y daños, incluyendo información sobre el uso del misoprostol. Una segunda consulta, una vez concluido el aborto, asegura a la mujer una evacuación completa, brindándole información sobre anticoncepción. En el momento del aborto mismo, la mujer está sola.

Si bien la normativa no contempla el aborto como un derecho de las mujeres, sino dentro de una praxis asistencial, ha contado con el apoyo de los grupos feministas, de derechos humanos y de jóvenes que ven en ella una posibilidad de ampliación de las prácticas médicas respecto a la interrupción voluntaria del embarazo en un marco de respeto hacia la mujer en situación de aborto. Constituye un cambio cualitativo de gran peso a nivel del imaginario y de la legitimidad que adquiere el tema en el espacio público. Por el contrario, su alcance sanitario es bajo, debido a la ausencia de medidas eficaces instrumentadas por el Ministerio de Salud Pública para su implementación.

Una segunda contradicción radica en que la normativa incluye brindar información sobre el uso del misoprostol; pero éste tiene la venta restringida, obteniéndose sólo con receta de gastroenterólogo, por lo que la mayoría de las mujeres que abortan lo obtienen en el mercado negro, lo que refuerza la clandestinidad del aborto.

En otro orden, contemporáneamente a los debates se ha fortalecido la noción de confidencialidad por parte de los equipos de salud, lo cual colabora a crear un clima de confianza con las mujeres en situación de aborto.

En lo que va del siglo, las discusiones parlamentarias se acompañaron por movilizaciones de los grupos anti-legalización del aborto que presionaban para que la legislación no se modifique, con el apoyo de la iglesia católica, especialmente los grupos ligados al Opus Dei, y de las iglesias neo-pentecostales. Por otro lado grupos y organizaciones feministas, de derechos humanos y de jóvenes desarrollaron diversas actividades, tanto para incidir en la opinión pública, como para presionar a los legisladores para que discutan el proyecto de ley tanto en el Senado como en la Cámara de Diputados y para lograr su aprobación.

Existe un desfase muy marcado entre los aspectos legislativos (legislación restrictiva), las políticas públicas correspondiente a la ordenanza 359/04 (estrategias de reducción de riesgo y daños de los abortos inseguros), y las prácticas de las mujeres (el aborto es un recurso generalizado para evitar maternidades forzadas). Los tres niveles recorren el espectro que va de sujetos abstractos, descorporizados a sujetos con nombre propio; muestran la complejidad de llevar a la práctica cotidiana los derechos sexuales y reproductivos.

En Argentina los procesos parlamentarios también parecen ser más lentos que los procesos observados en el campo de las políticas públicas (CESILINI et al., 2002). En este sentido vale destacar las acciones desde el Ministerio de Salud Pública tendientes a instaurar un servicio de atención pos aborto y de consejería en 2007 , el cual aún no ha podido implementarse de manera adecuada.

En otro orden, el movimiento feminista en Argentina ha desempeñado un papel central en colocar el tema en la agenda. Desde los inicios de los 80 un grupo de mujeres constituyeron la "Comisión por el derecho al aborto", desarrollando eficaces acciones en diferentes espacios políticos y sociales. En su momento se incluyeron en el grupo MADEL, pero surgieron en el desarrollo de las acciones dos posiciones: por un lado, quienes consideraban que los derechos sexuales y reproductivos "silenciaban" la cuestión del aborto y por el otro, quienes consideraban que los incluía. En las acciones mencionadas anteriormente prevaleció esta segunda posición. No obstante, dicha comisión ha persistido sistemáticamente hasta la actualidad en la lucha por la legalización del aborto y han presentado proyectos de ley en diversas oportunidades. Del mismo modo que se integraron en la Central de Trabajadores Argentinos, logrando introducir la legalización del aborto como un tema importante en las decisiones de la Central. 
Los acontecimientos del año 2001, la gran crisis política y económica llevó a un reacomodamiento de los movimientos sociales en el país. Allí las demandas, en general exclusivas de las mujeres, encontraron un nuevo espacio de expansión especialmente en las acciones desarrolladas en los movimientos de base y en las asambleas de barrio. El efecto de amplificación del tema de los derechos sexuales y reproductivos y el aborto permitió incorporar a la demanda a sectores y grupos sociales que permanecían alejados de esa problemática.

En 2005 se instaura la Campaña Nacional por el aborto, legal, seguro y gratuito, que por primera vez incorporó una dimensión federal. La Campaña se transforma en un interlocutor válido y reconocido de los espacios políticos, básicamente la Cámara de Diputados. El Día de la No Violencia contra la Mujer (2005) se realizó una marcha hacia el Congreso Nacional y se entregó el petitorio a favor de la despenalización y legalización del aborto de las 100.000 firmas recogidas. La marcha tenía la consigna "educación sexual para decidir, anticonceptivos para no abortar y aborto legal para no morir", lo cual implica la correspondiente "atención gratuita en los hospitales y obras sociales" y "el cumplimiento de la resolución ministerial sobre la atención humanizada del pos-aborto".

El documento propone "la conformación de una comisión tripartita, formada por organizaciones de mujeres y otras, y representantes de los poderes legislativo y ejecutivo" para elaborar un proyecto de ley. La declaración fue recibida por los legisladores. El impacto de la campaña va a producir presentaciones en el Congreso de proyectos para despenalizar, un debate importante dentro del Ministerio de Justicia sobre la reforma del código penal, sobre si entraba la cuestión del aborto o no, declaraciones de los dirigentes políticos y la presentación en el Congreso de la necesidad de organizar una comisión tripartita entre el gobierno, la legislatura y la sociedad civil para discutir la despenalización. Estas acciones han llevado a que el tema forme parte de la agenda pública y sobre todo de los medios masivos de comunicación, donde a través de diversos mecanismos se intenta relevar la opinión de la población. La mayoría de las encuestas de opinión pública revelan el apoyo de la ciudadanía sobre todo en la despenalización de los motivos que aún contempla punitivamente el código penal. (PETRACCI, 2007).

Con respecto a la segunda etapa de la campaña, la actividad mas importante fue la presentación en el Día Internacional de Acción por la Salud de las Mujeres en 2007, de una propuesta de proyecto de Ley consensuada hacia fines del año 2006, luego de un intenso trabajo de debates, consultas, asesoramientos, análisis de legislaciones comparadas, etc. El proyecto establece "la despenalización del aborto, salvo que se realice contra la voluntad de la mujer y la legalización del aborto voluntario, lo que significa que se realice por decisión de la mujer hasta las 12 semanas de gestación y sin límite de tiempo en los casos de violación, peligro de salud o vida y malformaciones fetales graves". (www. derechoalaborto.com.ar).

En los últimos años se han desplegado importantes denuncias a nivel del poder judicial, que han dado como resultado dictámenes que, al establecer jurisprudencia, son una herramienta importante para las acciones futuras, entre otras, eliminación -acorde a la legislación- del pedido de autorización judicial por parte de los médicos en los casos de aborto no punible.

Finalmente, cuando hablamos del aborto hay que considerar que lo controvertido de ello remite a un cuestionamiento radical del modo en que es pensado el orden social y el poder, dado que pone en escena la problemática de la discusión moral (y religiosa) y su deslizamiento hacia lo jurídico, interpela al orden patriarcal, remite a la inequidad de género, desnuda las problemáticas de la salud pública; reformula la dimensión de lo público y lo privado; explicita la escisión placer/reproducción, pone entre paréntesis el modelo de familia hegemónico, redefine la libertad de las mujeres para decidir sobre su destino y elecciones y sobre todo, revierte la lógica de una sexualidad normativa (GUTIÉRREZ, 2004a). 


\section{Referencias}

CEDES. Morbilidad materna severa en la Argentina. Estimación de la magnitud del aborto inducido. Resumen Ejecutivo, 2007. Disponible en <http://www.cedes.org/ informacion/ci/publicaciones/index.html>.

CELS; CLADEM; FEIM; ISPM. Derechos humanos de las mujeres: asignaturas pendientes del Estado Argentino. Contrainforme. Convención sobre la eliminación de todas las formas de discriminación contra la mujer (CEDAW). Buenos Aires, 2002.

CESILINI, S.; GHERARDI, N. Los límites de la ley. La salud reproductiva en la Argentina. Buenos Aires: Equipo de Género Región de América Latina y el Caribe, 2002.

CHECA, S.; ROSENBERG, M. Aborto hospitalizado. Buenos Aires: El cielo por asalto, 1996.

DOMÍNGUEZ, A.; SOLDEVILA A.; VÁZQUEZ, L.; ROSEMBERG, M.; PALMA. Z.; CHECA, S.; BIANCO, M.; GUTIÉRREZ M.A.; MARIÑO, A.; LASKI, M; ALANIS, M.; AMBACHS V.; JULIA S. Salud y aborto en Argentina: de las propuestas a los hechos. Córdoba, Argentina: SEAP, 2004

GOMES, E. C. A religião em discurso: a retórica parlamentar sobre o aborto. Relatório de pesquisa entre o público e o privado: a influência dos valores religiosos na tramitação de Projetos de Lei no Brasil. São Paulo: Prosare/CCR, 2007.

GRIMES, D. A.; BENSON, J.; SINGH, S.; ROMERO, M.; GANATRA, B.; OKONOFUA, F. E.; SHAH, I. H. Unsafe abortion: the preventable pandemic. Lancet, Nov. 25; 368(9550): 1908-19, 2006.

GUILLAUME, A.; LERNER, S. El aborto en América latina y el Caribe. Una revisión de la literatura de los años 1990 a 2005. París-México: Centre Population et Développement, 2007 (CD-ROM).

GUTIÉRREZ, M. A. Silencios y susurros: la cuestión de la anticoncepción y el aborto. Revista Jurídica Universidad Interamericana de Puerto Rico, San Juan de Puerto Rico, Facultad de Derecho, vol. XXXVIII, n. 1, Septiembre/Diciembre, 2003a.
Iglesia Católica y política en

Argentina: el impacto del fundamentalismo en las políticas públicas sobre sexualidad. Diálogos Sur-Sur sobre religión, derechos y salud sexual y reproductiva: los casos de Argentina, Colombia, Chile y Perú. Santiago de Chile, Universidad Academia de Humanismo Cristiano, 2004b, p.15-47.

Saberes de la opresión, saberes de la emancipación: la historia del movimiento de mujeres en la lucha por el aborto legal, ponencia presentada en $\mathrm{V}$ o Congreso CEISAL, Bruselas, 11/13 de abril 2007.

MINYERSKY, N. Derecho al aborto: nuevas perspectivas. Discurso pronunciado en la Asociación de abogados, 21 de junio 2005, mimeo.

MONTEIRO, M. F. G.; ADESSE. L. Estimativas de aborto induzido no Brasil e Grandes Regiões (1992-2005). In: ENCONTRO NACIONALDE ESTUDOS POPULACIONAIS, 15. Anais... Caxambu, Abep, 2006.

PETRACCI, M. Salud, derechos y opinión pública. Colômbia: Ed. Norma, 2007.

ROCHA, M. I. B. A discussão política sobre aborto no Brasil: uma síntese. Revista Brasileira de Estudos de População, São Paulo, v.23, n. 2, p. 369-374, jul./dez. 2006.

Planejamento familiar e aborto: discussões políticas e decisões no Parlamento. In: ÁVILA, M. B.; PORTELLA, A. P.; FERREIRA, V. (Orgs.). Novas legalidades e democratização da vida social: família, sexualidade e aborto. Rio de Janeiro: Garamond, 2005, p. 135-219.

A questão do aborto no Brasil: o debate no Congresso. Revista de Estudos Feministas, Rio de Janeiro, v.4, n.2, p. 381-98, 1996. (The abortion issue in Brazil: a study of the debate in Congress. Encarte no v. 4, n.2, p. 505-16, 1996).

ROCHA, M. I. B.; MORELL, M. G. G. A discussão política sobre a questão do aborto: um estudo comparado Brasil e Uruguai. In: CONGRESO DE LA ASOCIACIÓN LATINOAMERICANA DE POBLACIÓN, 2. Anais... 
Guadalajara: Alap, 2006. (disponível no CD-ROM do congresso).

ROCHA, M.I.B.; ANDALAFT NETO, J. A questão do aborto: aspectos clínicos, legislativos e políticos. In: BERQUÓ, E. (Org.). Sexo e vida: panorama da saúde reprodutiva no Brasil. Campinas: Editora Unicamp, 2003.

ROSTAGNOL, S. El conflicto mujer-embrión en el debate parlamentario sobre el aborto.

Revista de Estudos Feministas, 16 (2): 667-674, 2008.

Historias en el silencio: prostitución infantil y adolescente en Montevideo y área metropolitana. Montevideo: RudaUnicef, 2007.

. Panorama del aborto en Uruguay. Trabalho apresentado no Seminário Estudos sobre a Questão do Aborto em Países da América do Sul com Ênfase no Brasil. Campinas: Nepo/Unicamp, 2007.

Complicaciones post-aborto como una etapa del proceso del aborto: los distintos actores involucrados. En: Foro por los Derechos Reproductivos, Seminario Regional sobre Monitoreo de la Atención de las Complicaciones Post Aborto en Hospitales Públicos en Áreas Urbanas. Buenos Aires: 21-22 abril, 2003.

SANSEVIERO, R. et al. Barreras. Investigación y análisis sobre el acceso de las mujeres al derecho a decidir. Montevideo: Ruda-AUPF-IPPF/RHO, 2008.

SANSEVIERO, R. Condena, tolerancia y negación. El aborto en Uruguay. Montevideo: CIIIP, 2003.

SANTIN, M. A. V. Sexualidade e reprodução. Da natureza aos direitos: a incidência da Igreja Católica na tramitação do Projeto de Lei 20/91 - aborto legal e Projeto de Lei 1151/95 - união civil entre pessoas do mesmo sexo. Tese (Doutorado). Florianópolis, Programa Interdisciplinar em Filosofia e Ciências Humanas, Universidade Federal de Santa Catarina, 2005.

SAPRIZA, G. La despenalización del aborto a la luz de la eugenesia. Uruguay (19341938). Revista Fragmentos de Cultura, 12(6):1121-1139, 2002

TALIB, R.; CITELI, T. Serviços de aborto legal em hospitais públicos brasileiros. São Paulo: CDD-Br, 2005.

\section{Resumo}

\section{Aborto e Parlamento: um estudo sobre Brasil, Uruguai e Argentina}

O presente trabalho é um estudo comparativo entre Brasil, Argentina e Uruguai em torno das legislações e ações de diversos atores em relação à descriminalização e/ou legalização do aborto. Este tema entrou na agenda pública dos três países a partir do processo de democratização, iniciado na década de 1980, e de diversas intervenções, tanto do movimento de mulheres, como da classe médica e outros atores sociais, ao longo da década de 1990 e dos primeiros anos do século XXI. Nesse sentido, foram estudadas as diversas intervenções no âmbito legislativo (tanto nos Senados como nas Câmaras de Deputados), bem como as estratégias das políticas públicas implementadas pelos Executivos, com monitoramento e acompanhamento do movimento de mulheres, visando enfrentar uma gravíssima situação de violação dos direitos humanos. O desrespeito aos direitos, consagrados nas Constituições, atinge as mulheres, em especial as mais vulneráveis, no tocante ao direito à saúde e à equidade. Além disso, foram analisados os avanços e retrocessos observados ao longo do período estudado, bem como a importância de se manter o tema na agenda pública e na mídia, que desempenhou um papel importante, tanto promovendo como deslegitimando o direito ao aborto.

Palavras-chave: Aborto. Ação parlamentar. Políticas públicas. Movimento de mulheres. Atores sociais. 


\begin{abstract}
Abortion and Parliament: a study on Brazil, Uruguay and Argentina

This article consists of a comparative study among three countries, namely, Brazil, Argentina and Uruguay, concerning their legislations and the participation of many different actors regarding the decriminalization and/or legalization of abortion.This topic entered the public agenda of all three countries as part of their processes of democratization, which began in the 1980s. It also involved a number of other interventions, such as action by women's movements and positions taken by physicians' associations and other social actors during the 1990s and the first years of the 21st century. A number of interventions in the legislative sphere were studied (in the countries' senates and chambers of deputies), as well as strategies for public policies implemented by the executive branches, monitored by women's movements as they faced serious violations of human rights. The disrespect of such rights, consolidated in the constitutions of the three countries, especially affects women, who are the most vulnerable regarding rights to health and equality. In addition to inroads and setbacks during the period studied, the author also studied the importance of keeping the topic on the public agenda and in the media during the period mentioned above. The media played an important role both in promoting and de-legitimizing the right to abortion.
\end{abstract}

Keywords: Abortion. Congressional action. Public policies. Social actors

Recebido para publicação em 07/10/2009. Aceito para publicação em 18/11/2009. 